\title{
Is there a Glass Transition in Planar Vortex Systems?
}

\author{
Thorsten Emig and Simon Bogner \\ Institut für Theoretische Physik, Universität zu Köln, Zülpicherstr. 77, 50937 Köln, Germany
}

(Dated: November 20, 2018)

\begin{abstract}
The criteria for the existence of a glass transition in a planar vortex array with quenched disorder are studied. Applying a replica Bethe ansatz, we obtain for self-avoiding vortices the exact quenched average free energy and effective stiffness which is found to be in excellent agreement with recent numerical results for the related random bond dimer model [1]. Including a repulsive vortex interaction and a finite vortex persistence length $\xi$, we find that for $\xi \rightarrow 0$ the system is at all temperatures in a glassy phase; a glass transition exists only for finite $\xi$. Our results indicate that planar vortex arrays in superconducting films are glassy at presumably all temperatures.
\end{abstract}

The existence of a vortex glass (VG) in type-II superconductors is of crucial importance for their performance. Quenched disorder affects both the translational order of the Abrikosov vortex lattice and the low current response [2]. The thermodynamic response of a planar VG in a mesoscopic superconducting film [3] has been studied in a recent experiment [4], providing 'fingerprints' of the sample's disorder [5]. Glassy phases are expected to be generic to a wide class of disorder dominated systems. The planar VG is a famous starting point within this class since it is among the very few systems where analytical progress seems possible. The situation is here analogous to that of exactly solvable 1D quantum systems which have advanced the understanding of strongly correlated systems.

Thermal fluctuations compete against the VG, raising the question of the existence of a glass transition at a finite temperature $T_{g}[\overline{6} \mid$. However, the literature on this subject is rather contradictory [1, 7, 8]. Major support, both analytical and numerical, for a finite $T_{g}$ stems from the phenomenologically related random field (RF) XY model which has a finite $T_{g}$ transition [9, 10]. However, considering the entropic vortex interaction, the absence of a finite $T_{g}$ transition has been predicted under certain assumptions as well [8]. The theory underlying the vortex system can be applied to a broad class of other systems like surface steps [11], polymers [12] and domain walls [13. A more fundamental aspect of the planar VG is related to its often debated replica description, including the concept of replica symmetry breaking, and the non-trivial exchange of replica and thermodynamic limit. Due to the relation to the recently simulated random bond dimer model [1], the VG provides a unique system to test replica theory and could be useful in clarifying important concepts in the statistical mechanics of disordered systems.

In this paper, we analyze the conditions under which a planar vortex lattice undergoes a finite $T_{g}$ glass transition. For vanishing vortex persistence length $\xi$, we find the lattice to be glassy at all temperatures with an analytic free energy. Analyticity of the free energy is also obtained for the vortex-free RFXY model. Our replica symmetric Bethe ansatz results are found to be in excel- lent agreement with recent simulations [1]. For finite $\xi$, there exists a finite $T_{g}$ which, however, is presumably too large to be observable in superconducting films.

Vortex system. - We consider a lattice of self-avoiding directed elastic lines of density $\rho=1 / a$ in $1+1$ dimensions. Each line is characterized by its position $x_{j}(z)$ and line tension $g$. The lines interact via a short-ranged repulsive pair potential $U(x)$. Quenched disorder couples locally to the lines via the random potential $V(\mathbf{r})$, $\mathbf{r}=(x, z)$, which is assumed to have short-ranged correlations $\overline{V(\mathbf{r}) V\left(\mathbf{r}^{\prime}\right)}=\Delta \delta\left(\mathbf{r}-\mathbf{r}^{\prime}\right)$. The total energy reads

$H=\int d z \sum_{j}\left\{\frac{g}{2}\left(\frac{d x_{j}}{d z}\right)^{2}+\sum_{i, i \neq j} U\left(x_{i}-x_{j}\right)+V\left(x_{j}, z\right)\right\}$.

This model can be considered as the continuum version of lines placed on the bonds of a rectangular lattice [14]. Below, we will also consider lines with a finite persistence length $\xi$, corresponding to correlated random walks in the lattice model.

Random field XY model. - The line lattice is usually treated within a $2 \mathrm{D}$ elastic theory for the displacement field $u(\mathbf{r})$ so that the line positions are $x_{j}(z)=j a+$ $u(j a, z)$. The effective Hamiltonian is then

$$
H_{\mathrm{XY}}=\int d^{2} r\left\{\frac{c_{11}}{2}\left(\partial_{x} u\right)^{2}+\frac{c_{44}}{2}\left(\partial_{z} u\right)^{2}+\rho(\mathbf{r}) V(\mathbf{r})\right\}
$$

with compression modulus $c_{11}=a U^{\prime \prime}(a)$, tilt modulus $c_{44}=g / a$ and line density $\rho(\mathbf{r})=\sum_{j} \delta\left(x-x_{j}(z)\right)$. Rewriting $\rho(\mathbf{r})$ as a Fourier series in $u(\mathbf{r})$, the model becomes equivalent to the random field XY model without vortices [6]. It is well known that, for weak disorder, this model shows a phase transition to a glassy phase at a finite temperature $T=T_{g}$. A renormalization group (RG) analysis yields the scaling dimension of disorder [9],

$$
\lambda_{\Delta}=2\left(1-\frac{\pi T}{a^{2} \sqrt{c_{11} c_{44}}}\right),
$$

giving $T_{g}=a^{2} \sqrt{c_{11} c_{44}} / \pi$. An important property of the $\mathrm{XY}$ model is that the elastic moduli remain unrenormalized due to a statistical symmetry [7, 9]. Thus, naively 
one would expect for the line lattice a transition at a $T_{g}$ determined by the elastic moduli on the length scale where $H_{X Y}$ becomes applicable. However, as we will show below, there is, in fact, a renormalization of $c_{11}$ in the original model of Eq. (11) which can even destroy the transition.

What are the manifestations of the glass transition in the XY model? Anomalous variations in the response to an external tilt of the lines have been used to characterize the glassy phase [7]. However, signatures of the transition in the free energy itself have not been studied so far. Using the known mapping of the XY model onto a vector Coulomb gas [9] one can derive the RG flow of the free energy as shown in 15] for the pure XY model. From this analysis we find that the quenched average free energy is analytic at $T_{g}$. Weak disorder makes the contribution

$$
f_{\Delta}=-\frac{\Delta}{T a^{2}}\left(1+\frac{\Delta}{c_{11}^{3 / 2} c_{44}^{1 / 2} a^{4}} \frac{\pi^{3}}{(1-\tau)^{2}(1-2 \tau)}\right)
$$

to the free energy density for small $\tau=1-T / T_{g}$, where we have neglected $T$-independent terms.

Another signature of the glassy phase are its correlations [2]. For $T>T_{g}$, one finds $\left[\left\langle(u(\mathbf{r})-u(\mathbf{0}))^{2}\right\rangle\right] \sim$ $\log (|\mathbf{r}| / a)$, and in the glassy phase for small $\tau>0$ the slightly faster growth $\left[\left\langle(u(\mathbf{r})-u(\mathbf{0}))^{2}\right\rangle\right] \sim \log ^{2}(|\mathbf{r}| / a)$. Here $\langle\ldots\rangle$ and $[. .$.$] denote thermal and disorder average,$ respectively. The decay of the corresponding densitydensity correlations suggests that the correlation length is infinite on both sides of the transition which is consistent with the predicted analyticity of free energy. Next we will focus on the correlations of thermal fluctuations $\delta u(\mathbf{r})=u(\mathbf{r})-\langle u(\mathbf{r})\rangle$ around the state pinned by disorder. On both sides of the transition one has

$$
C(\mathbf{r})=\left[\left\langle(\delta u(\mathbf{r})-\delta u(\mathbf{0}))^{2}\right\rangle\right]=\frac{a^{2}}{16 \pi K} \log (|\mathbf{r}| / a),
$$

with $K=(\pi / 16) T_{g} / T=\left(a^{2} / 16\right) \sqrt{c_{11} c_{44}} / T$. Below, we will calculate $K$ directly in terms of the parameters of the original model of Eq. (1).

Self-avoiding vortices. - A generic but simpler model is obtained if the only vortex interaction of Eq. (11) is the one representing the non-crossing condition, i.e., a hardcore repulsion $U(x)=c \delta(x)$ with $c \rightarrow \infty$. Using the replica method, with $n$ replicas, the model can be mapped to $S U(n)$ fermions and thus can be solved $e x$ actly by a replica Bethe ansatz (RBA) without replicasymmetry breaking [14. To date, only deep inside the glassy phase, e.g., for $H \rightarrow H_{c 1}$, the quenched average free energy density $[f]$ has been studied by this technique [14, 16]. However, at arbitrary $H$ (or density $\rho$ ) the RBA yields

$$
[f]=f_{0} \rho+\frac{T^{2}}{2 g} \int_{-Q}^{Q} q^{2} \varrho(q) d q
$$

where $f_{0}$ is the single vortex free energy per unit length; its precise form is unimportant in what follows. $\varrho(q)$ is determined by

$$
\int_{-Q}^{Q}\left\{\frac{1}{l_{d}\left(q-q^{\prime}\right)}+\pi \operatorname{coth}\left(\pi l_{d}\left(q-q^{\prime}\right)\right)\right\} \varrho\left(q^{\prime}\right) d q^{\prime}=q
$$

with length scale $l_{d}=T^{3} / g \Delta$, and $Q$ is fixed by $\int_{-Q}^{Q} \varrho(q) d q=\rho$. The integral equation $(\mathbb{7})$ can be solved perturbatively in $Q l_{d}$, leading to

$$
\varrho(q)=\sqrt{1-(q / Q)^{2}}\left[\frac{1}{2 \pi} Q l_{d}-\frac{\pi}{24}\left(Q l_{d}\right)^{3}+\ldots\right] .
$$

Higher order terms can be easily calculated and will depend also on $q$, thus changing the functional form of the lowest order result. In the opposite limit, $Q l_{d} \rightarrow \infty$, i.e, for vanishing disorder, the solution is $\varrho(q)=1 /(2 \pi)$. With the result for $\varrho(q)$ at hand, both $\rho l_{d}$ and the integral of Eq. (6) can be obtained as a series in $\left(Q l_{d}\right)^{2}$. However, we are actually interested in eliminating $Q$ in order to calculate $[f]$ in terms of $\rho$ and $l_{d}$. At this stage, a particularly interesting property of $\varrho(q)$ is important. The exact result for the integral of Eq. (6) as a function of $\rho l_{d}$ is obtained from the two lowest order terms of Eq. (8). Indeed, it can be shown order by order in $Q l_{d}$ that the exact result is $\int_{-Q}^{Q} q^{2} \varrho(q) d k=\frac{\pi^{2}}{3} \rho^{3}+\rho^{2} l_{d}^{-1}$. This result we could also confirm numerically with high precision. Thus, the RBA gives for the quenched average free energy density the exact result

$$
[f]=f_{0} \rho+\frac{\pi^{2}}{6} \frac{T^{2}}{g} \rho^{3}+\frac{\Delta}{2 T} \rho^{2} .
$$

For the interaction (last two) terms it is possible to give a simple explanation. The first is just the known Pokrovski-Talapov interaction 13] from entropy loss due to collisions of thermally fluctuating lines in pure systems. The second term can be explained by a similar argument which gives the interaction in the dilute limit $\rho \ll l_{d}^{-1}$. It arises from an increase in energy due to collisions which prevent the lines from gaining their optimal energy from the random potential [14]. It is rather unexpected that the total effective interaction is just the sum of these two contributions. As a consequence of that, the free energy is an analytic function. Based on our result for the XY model, this does not exclude the possibility of a finite- $T$ glass transition. However, its existence can be excluded by computing the scaling dimension $\lambda_{\Delta}$, Eq. (3), in terms of the macroscopic compression modulus $c_{11}^{0}$. It is given by the effective interaction, $c_{11}^{0}=a U_{\text {eff }}^{\prime \prime}(a)$, with $U_{\text {eff }} / a$ corresponding to the two last terms of Eq. (9). Using $c_{44}=g / a$, and

$$
c_{11}^{0}=\frac{\pi^{2} T^{2}}{g a^{3}}+\frac{\Delta}{T a^{2}},
$$

we find $\lambda_{\Delta}>0$ at all finite temperatures, i.e., $T_{g}=\infty$. With the exact $c_{11}^{0}$ available, we are able to relate the 
phenomenological stiffness $K$ of Eq. (5) to the microscopic parameters of the model of Eq. (1), leading to

$$
K=\frac{\pi}{16}\left(1+\frac{a g \Delta}{\pi^{2} T^{3}}\right)^{1 / 2}
$$

For vanishing disorder, $\Delta=0$, the previously known result $K=\pi / 16$ is recovered 17 .

There are a few comments in order to clarify the apparent discrepancy between the exact RBA results for self-avoiding lines and the RG predictions for the random field XY model of Eq. (2). To begin with, on microscopic length scales there is no direct link between the two models since $c_{11}=0$ due to self-avoidance as only interaction. However, the XY model is generally believed to describe the vortex lattice at length scales larger than $a$ where a finite fluctuation induced $c_{11}$ exists [6, 8]. That this point of view is incorrect for finding out about the existence of a glass transition in the vortex system shows the following argument. On large but finite length scales $L \gg a^{2} g / T$ the effective $c_{11}$ for $\Delta \rightarrow 0$ can be obtained from the analogy of the self-avoiding vortex lines to $1 \mathrm{D}$ fermions [14]. Since a finite vortex length $L$ translates into a finite temperature for the fermions, the Sommerfeld expansion can be used to get the correction $\delta c_{11}=-a g / 3 L^{2}$ to the result of Eq. (10). Thus, on any sufficiently large length scale, disorder seems to be irrelevant since then $\lambda_{\Delta}<0$. In contrast, the RBA shows that disorder is always relevant. The contradicting results are explained by the fact that the statistical symmetry in the XY model is broken on all length scales by the non-crossing condition.

Dimer model. - The self-avoiding vortices can be simulated directly, i.e., not via the XY model, using a mapping to a discrete dimer model with quenched bond disorder [18]. A recently developed polynomial algorithm allows to study large systems at finite temperature but fixed density $\rho=1 / 2$ (in units of the dimer lattice constant) [1]. Comparison of our RBA predictions with the results of this numerical approach provides a critical test of the replica approach, including replicasymmetry and proper analytical continuation while taking the number of replicas to zero. The dimer model is defined as follows: Choose a subset (whose elements are called dimers) of the bonds on a square lattice, such that every lattice site is touched by exactly one of these dimers. The energy of one such dimer covering $D$ is defined by $H_{d}=\sum_{(i, j) \in D} \epsilon_{i j} / T_{d}$, where the sum is over all dimers of $D, \epsilon_{i j}$ are random bond energies drawn from a Gaussian distribution with zero mean and a variance of one, and $T_{d}$ is the dimer temperature. Via the wellknown mapping to the solid-on-solid model, it is possible to assign every dimer covering a height function which maps to the line displacement $u(\mathbf{r})$ in the vortex system representation. This representation results from the superposition of an arbitrary covering $D$ and a fixed reference covering that specifies the direction of the lines, cf. Ref. [1]. By measuring the height configurations, Zeng et al. could determine the correlation function of Eq. (5), and thus the stiffness $K$ as a function of $T_{d}$ [1]. In order to compare their results to our prediction of Eq. (11), the parameters $g / T$ and $\Delta / T^{2}$ have to be determined for the dimer representation of the vortex system. It is easy to see that the ratio of horizontal and vertical line steps on the lattice yields $g / T=2$. The precise relation between $\Delta$ and $T_{d}$ can be obtained by comparing the annealed average free energy of the continuum model of Eq. (1) and the discrete dimer version of the vortex lattice. Using known methods for computing the partition function of dimer models [19], we get

$$
\frac{\Delta}{\xi_{d} T^{2}}=\frac{2}{T_{d}^{2}}-\frac{4 G}{\pi}+\frac{4}{\pi} \int_{0}^{e^{-T_{d}^{-2} / 2}} d x \frac{\arctan (x)}{x},
$$

with $\xi_{d}$ a regularization length for the disorder correlator [20], and Catalan's constant $G=0.915966$. With the above relations and $a=1 / \rho=2$ in Eq. (11), we obtain $K\left(T_{d}\right)$ as plotted in Fig. 1, together with the data provided by the dimer algorithm of Ref. [1]. Obviously, there is excellent agreement between our RBA results and simulations.

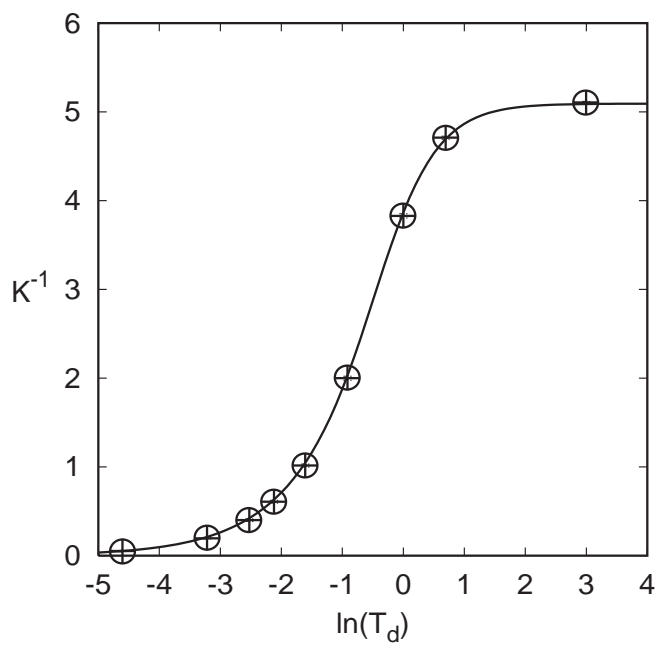

FIG. 1: Replica Bethe ansatz result for the stiffness $K$, Eq. (11), plotted as function of the dimer temperature $T_{d}$, using Eq. (12) with $\xi_{d}=1.15$, and the corresponding data (circles) from the dimer model algorithm of Ref. [1].

Finite interaction and persistence length. - So far, we have shown that the model of Eq. (11) with a vanishing single line persistence length $\xi$ and $U(x)$ representing only a non-crossing condition is always in the glassy phase. We now consider the effects of an arbitrary repulsive interaction $U(x)$ and a finite persistence length $\xi$. For the vortex system, $U(x)$ diverges for $x \rightarrow 0$ and decays exponentially for large $x ; \xi$ can be identified with the coherence length of the superconductor.

Let us first discuss a general interaction while keeping $\xi=0$. The relevance of disorder now depends on the 
interaction induced change in $c_{11}$ for $\Delta \rightarrow 0$, cf. Eq. (3). This change can be obtained by employing the known mapping 13 of elastic lines to 1D spin-less fermions which however interact via $U(x)$, denoting now the finite distance interaction on top of the self-avoidance. The compressibility $\kappa$ of the fermions is related to the compression modulus of the vortex system according to $1 / \kappa=a^{2} c_{11}$. For weak interaction, $\kappa$ is easily calculated, e.g., by bosonization. Using the standard notation of Luttinger liquid theory [21], one gets $\kappa=1 / \pi \hbar v_{N}$ with $v_{N}=v_{F}+(\tilde{U}(0)+\tilde{U}(2 \pi / a) / 2) / \hbar \pi$, where $\tilde{U}(q)$ is the Fourier transform of $U(x)$. Thus, any repulsive interaction increases the effective compression modulus $c_{11}=c_{11}^{\mathrm{PT}}+c_{11}^{\text {int }}$ with

$$
c_{11}^{\mathrm{int}}=\frac{1}{a^{2}}\left(\tilde{U}(0)+\frac{1}{2} \tilde{U}(2 \pi / a)\right),
$$

and for $U(x) \rightarrow 0$, the known Pokrovsky-Talapov result $c_{11}=c_{11}^{\mathrm{PT}}=\pi^{2} T^{2} / g a^{3}$ is recovered. Thus according to Eq. (3), the scaling dimension of disorder is increased by any finite vortex interaction, and the system remains always in the glassy phase.

Next, we neglect interactions for the time being and now consider the thermally fluctuating vortex lines as correlated random walks, i.e., we assume a finite persistence length $\xi$. These correlations increase the fluctuations of a single line. Denoting the so decreased macroscopic line tension again by $g$, entropy reduction due to line collisions leads not only to the Pokrovsky-Talapov result but also to an additional renormalization of the modulus $c_{11}=c_{11}^{\mathrm{PT}}-c_{11}^{\xi}$ where, for $\xi \ll a^{2} g / T$,

$$
c_{11}^{\xi}=\frac{10}{3} \frac{T \xi}{a^{2} g} c_{11}^{\mathrm{PT}} .
$$

For larger $\xi, c_{11}$ increases relative to this first order result. Including the effect of disorder on $c_{11}$, cf. Eq. (10), one obtains from Eq. (3) the finite transition temperature

$$
T_{g}=0.42\left(\frac{g^{2} \Delta}{\rho^{3} \xi}\right)^{1 / 4} .
$$

Thus, a finite persistence length yields a finite temperature glass transition.

Finally, we study the combined effect of interactions and a finite persistence length. If disorder is strong compared to the interaction, $\Delta \gg T(\tilde{U}(0)+\tilde{U}(2 \pi / a) / 2)$, the result of Eq. (15) remains applicable. In the opposite limit, $T_{g}$ is independent of disorder,

$$
T_{g}=0.31\left(\frac{g^{2}(\tilde{U}(0)+\tilde{U}(2 \pi / a) / 2)}{\rho^{3} \xi}\right)^{1 / 3} .
$$

For vanishing persistence length, $T_{g}$ diverges, and the system is glassy at all temperatures.

Discussion. - The model of Eq. (1) applies to vortex systems in films of thickness $d \lesssim a, \lambda$ with $\lambda$ the penetration depth. Then the crucial questions is if the glass transition temperature $T_{g}$ can be smaller than the critical temperature $T_{c}$ of the superconductor. Since the only mechanism leading to a finite $T_{g}$ is a finite persistence length, we maximize the effect from $c_{11}^{\xi}$ in Eq. (14) by considering small $a \ll \lambda$. Then GinzburgLandau theory yields a $\tilde{U}(q)[3]$ which is too large for the bosonization result of Eq. (13) to be valid. However, a lower bound is $c_{11}^{\text {int }}>b c_{11}^{\mathrm{PT}}$ with $b$ of order one. Using Eq. (14), we find $T_{g}>3 b / 10 \times a^{2} g / \xi$. With the stiffness $g=\Phi_{0}^{2} / 16 \pi \times d / a \lambda^{2}, \Phi_{0}=h c / 2 e$, [3], we obtain in the limit of infinitesimal disorder $T_{g} \gtrsim \Phi_{0}^{2} / 16 \pi^{2} \times a d / \xi \lambda^{2}$ which becomes, with $a, d>\xi, T_{g} / T_{c}>1-5 \cdot 10^{-4}$ for $T_{c}=100 \mathrm{~K}$ and with the length $\xi_{0}=10 \AA, \lambda_{0}=100 \AA$ at $T=0$. Thus, $T_{g}$ is extremely close to $T_{c}$, and presumably any small amount of quenched disorder will raise $T_{g}$ above $T_{c}$, making it impossible to observe a glass transition in planar vortex systems.

We thank M. Kardar, T. Nattermann, S. Scheidl and C. Zeng for useful discussions, and especially C. Zeng for sending the data of Fig. 1. This work was supported by DFG through Emmy-Noether grant No. EM70/2-1.

[1] C. Zeng, P. L. Leath, and T. Hwa, Phys. Rev. Lett. 83, 4860 (1999).

[2] T. Nattermann and S. Scheidl, Adv. Phys. 49, 607 (2000).

[3] A. A. Abrikosov, Soviet Phys. JETP 19, 988 (1964).

[4] C. A. Bolle et al., Nature 399, 43 (1999).

[5] T. Emig and M. Kardar, Phys. Rev. Lett. 85, 2176 (2000).

[6] M. P. A. Fisher, Phys. Rev. Lett. 62, 1415 (1989).

[7] T. Hwa and D. S. Fisher, Phys. Rev. Lett. 72, 2466 (1994).

[8] T. Nattermann, I. Lyuksyutov, and M. Schwartz, Europhys. Lett. 16, 295 (1991).

[9] J. L. Cardy and S. Ostlund, Phys. Rev. B 25, 6899 (1982).

[10] T. Giamarchi and P. Le Doussal, Phys. Rev. Lett. 72, 1530 (1994).

[11] W. W. Mullins and R. F. Sekerka, J. Appl. Phys. 35, 444 (1964).

[12] P. G. de Gennes, J. Chem. Phys. 48, 2257 (1968).

[13] V. L. Pokrovsky and A. L. Talapov, Phys. Rev. Lett. 42, 65 (1979).

[14] M. Kardar and D. R. Nelson, Phys. Rev. Lett. 55, 1157 (1985).

[15] J. M. Kosterlitz, J. Phys. C 7, 1046 (1974).

[16] T. Emig and M. Kardar, Nucl. Phys. B 604, 479 (2001).

[17] C. L. Henley, J. Stat. Phys. 89, 483 (1997).

[18] C. Zeng, A. A. Middleton, and Y. Shapir, Phys. Rev. Lett. 77, 3204 (1996).

[19] P. W. Kasteleyn, Physica (Utrecht) 27, 1209 (1961).

[20] The regularization length $\xi_{d}$ must appear here since a discrete and a continuum model are related.

[21] J. Voit, Rep. Progr. Phys. 58, 977 (1995). 